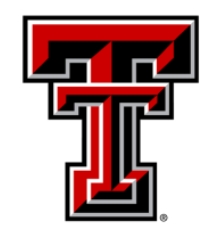

TEXAS TECH UNIVERSITY

Libraries"

\title{
NUMERICAL EVALUATION OF HYDROGEN OUTGASSING FROM COPPER ELECTRODES WITH MITIGATION BASED ON A TUNGSTEN CAPPING LAYER
}

\section{The Texas Tech community has made this publication openly available. Please share how this access benefits you. Your story matters to us.}

\begin{tabular}{|c|c|}
\hline Citation & $\begin{array}{l}\text { J. Acharjee and R. P. Joshi, "Numerical Evaluation of Hydrogen } \\
\text { Outgassing from Copper Electrodes with Mitigation Based on a } \\
\text { Tungsten Capping Layer," Physics of Plasmas 26, } 093504 \text { (2019). } \\
\text { https://doi.org/10.1063/1.5109682 }\end{array}$ \\
\hline Citable Link & https://hdl.handle.net/2346/86901 \\
\hline Terms of Use & $\begin{array}{l}\text { This article may be downloaded for personal use only. Any other } \\
\text { use requires prior permission of the author and AIP Publishing. }\end{array}$ \\
\hline
\end{tabular}




\section{Numerical evaluation of hydrogen outgassing from copper electrodes with mitigation based on a tungsten capping layer}

Cite as: Phys. Plasmas 26, 093504 (2019); https://doi.org/10.1063/1.5109682

Submitted: 10 May 2019. Accepted: 17 August 2019. Published Online: 06 September 2019

J. Acharjee, and $(\mathbb{D}$ R. P. Joshi

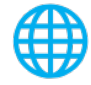

\section{ARTICLES YOU MAY BE INTERESTED IN}

Probing changes in secondary electron yield from copper electrodes due to surface defects and changes in crystal orientation

Journal of Applied Physics 126, 123301 (2019); https://doi.org/10.1063/1.5113642

Plasma flow equilibria in 2D cylindrically symmetric expanding magnetic field Physics of Plasmas 26, 093501 (2019); https://doi.org/10.1063/1.5090559

An empirical approach for parameters estimation of underwater electrical wire explosion Physics of Plasmas 26, 093502 (2019); https://doi.org/10.1063/1.5111518 


\title{
Numerical evaluation of hydrogen outgassing from copper electrodes with mitigation based on a tungsten capping layer
}

Cite as: Phys. Plasmas 26, 093504 (2019); doi: 10.1063/1.5109682

Submitted: 10 May 2019 - Accepted: 17 August 2019 .

Published Online: 6 September 2019

J. Acharjee and R. P. Joshi ${ }^{\text {a) }}$ (D)

\author{
AFFILIATIONS \\ Department of Electrical and Computer Engineering, Texas Tech University, Lubbock, Texas 79409, USA
}

a) Author to whom correspondence should be addressed: ravi.joshi@ttu.edu

\begin{abstract}
Outgassing remains a pertinent issue for high power applications and is exacerbated by the high field driven, localized heating environments commonly encountered. Here, molecular dynamics simulations are performed for a simple model-based assessment of outgassing from electrodes. Our results of temperature dependent diffusion coefficients for hydrogen in copper agree well with experimental reports over a wide range spanning $300 \mathrm{~K}$ to $1330 \mathrm{~K}$. Separate results are also obtained for transport of hydrogen to ascertain whether a grain-boundary would facilitate channeled transport or work to impede flow by clustering the gas atoms. Finally, the use of a tungsten overlayer on copper is evaluated as a material-based strategy for mitigating outgassing. It is demonstrated that a few monolayers of tungsten coating on the outer surface can be effective in significantly reducing outdiffusion at $700 \mathrm{~K}$.
\end{abstract}

Published under license by AIP Publishing. https://doi.org/10.1063/1.5109682

\section{INTRODUCTION}

High power devices operating under high voltage conditions are used in numerous applications including vacuum electronics, ${ }^{1-3}$ particle acceleration, ${ }^{4,5}$ inertial confinement fusion, ${ }^{6}$ and microwave generation. ${ }^{2}$ The high electric fields (typically exceeding $100 \mathrm{kV} / \mathrm{cm}$ ) are strong drivers for electron emission and also cause localized heating at electrodes, explosive material vaporization, and/or desorption of gases. ${ }^{7,8}$ Gas desorption from materials can be an important factor in plasma formation in such systems, which can then lead to breakdown or surface flashover. Apart from plasma formation, outgassing has also been known to lead to anode-cathode gap closure and pulse shortening. ${ }^{10}$ The interactions of emitted electrons and gas molecules, both of which are released by the impact of high energy electrons on the electrode surface, ${ }^{11,12}$ support local heating of the electrodes, ${ }^{8}$ thus working to facilitate and strengthen outgassing. ${ }^{13}$

In this high-power context, Neuber et al. ${ }^{12}$ had established the role of outgassing through time-resolved spectroscopy which revealed the existence of excited atomic hydrogen, a species with the highest mobility due to its small mass. Hydrogen outgassing has also been reported from several metals. ${ }^{14}$ In the context of pulsed power devices, the monolayers of gas atoms that are usually adsorbed on the surface are likely to be liberated by direct electron impact. This can have downstream consequences such as the providing an additional source of neutrals for ionization and space-charge development. From an electrical standpoint, the operating conditions can then become dynamic and affect the efficiencies of the operating devices, as pointed out by Haworth et al. ${ }^{15}$ Information of such outgassing could, for example, be fed into particle-in-cell calculations for a more comprehensive analysis of High Power Microwave devices. This phenomenon of gas desorption is typically observed through an increase in residual gas pressure. ${ }^{16,17}$ Besides influencing breakdown physics and high voltage device operation, trapped gases in metal electrodes can also adversely affect the material properties, degrade mechanical strength, and contribute to embrittlement. ${ }^{18-22}$ In any event, while the role of outgassing and gas emission from surfaces subject to high electric fields is well established, there have not been many studies on the gas release dynamics or assessments of potential mitigation techniques, especially taking account of the inherent microscopic details. For example, it is not clear whether the use of suitable cap-layers might help reduce outgassing, and if so, to what degree, nor have there been model-based assessments on particular materials that might be most suited for such reductions in outgassing.

In this contribution, we present the results of hydrogen diffusion in copper based on molecular dynamics (MD) simulations. The technique is general, and copper has been chosen as a representative metal that is commonly used in high voltage applications. This study is 
driven by a need to quantify and assess potential mitigation of outgassing for pulsed-power applications. The microscopic MD technique includes many-body effects, enables complex atomic-scale structures (such as grain boundaries and defects) to be accurately replicated in the computational scheme, natural factors in internal energy barriers, and allows for the inclusion of thermal and/or density gradients as drivers for internal transport. Here, hydrogen diffusion in a perfect face-centered-cubic (fcc) copper lattice is first simulated at different temperatures and compared with numerous data reported in the literature to test model validity. Separate results are then obtained for transport of atomic hydrogen in the presence of grain boundaries to probe whether a grain-boundary would facilitate channeled transport or work toward the clustering of gas atoms. Finally, the use of a bodycentered cubic (bcc) tungsten overlayer on copper is evaluated as a material-based strategy for reducing hydrogen outgassing. It is demonstrated that a few monolayers of tungsten coating on the outer surface can be effective in significantly reducing outdiffusion.

The overall scope of such studies is vast, can potentially include a variety of defects (such as defects, twin boundaries, dislocations, and network of grain boundaries), and could even include dynamic changes in the material structure such as gas accumulation and bubble formation $^{23}$ or the dynamic temperature increase arising from the energy expended by incoming charged entities striking the electrodes. However, the present scope is really a more modest attempt and a simple step in applying the Molecular Dynamics (MD) technique to probe a simple situation of outgassing from copper at different temperatures, along with the inclusion of a grain boundary and assessments of outgassing mitigation through the use of a tungsten cap-layer.

\section{MODEL DETAILS}

Hydrogen diffusion in a copper lattice was simulated using the classical molecular dynamics (MD) scheme. In this technique, forces on any atom at a lattice site due to its many neighbors are evaluated based on semiempirical interatomic potentials. In a sense, this approach allows one to pursue computational experiments by probing a variety of different configurations and to include several species with their unique interactions at the molecular level. Its strength includes the ability to study fast nonequilibrium processes with an atomic-level resolution. The limitations of the technique are well known. These include the inability to explicitly include electrons, the introduction of potential energy surfaces that are a function of atomic positions only (i.e., the Born-Oppenheimer approximation), and the treatment of potential energy surfaces through analytical functions that provide information as a function of atomic coordinates. Also, most approaches are based on classical Newtonian mechanics and ignore quantum effects and corrections. Furthermore, the computational complexity usually restricts the simulation length scales to tens or hundreds of nanometers and time durations to the nanosecond regime. As a result of the finite size restrictions, deviations from the actual boundary conditions could also result.

Nonetheless, such simulations are a useful tool for analyzing and predicting defect properties, phase transformation mechanisms, kinetics and resultant structural evolutions, and dynamic behaviors of materials. ${ }^{24,25}$ Since the results can depend on the interatomic potentials chosen, a brief review is perhaps in order. Several empirical potentials have been proposed such as the Embedded Atom Method $(\mathrm{EAM})^{26}$ and Finnis-Singlair, ${ }^{27}$ Glue, ${ }^{28}$ Tersoff, $^{29}$ and the RGL ${ }^{30}$ models for metallic or covalent hard-material systems. Bond order potentials (BOPs), which include the Finnis-Singlair and Tersoff formulations, are a class of empirical, analytical interatomic potentials that are often used and have the advantage of describing several different bonding states of an atom with the same parameters. The early version of the BOP potential ${ }^{31}$ was improved to include distinct bond orders for sigma and pi-bonds. ${ }^{32}$ Other developments include the adaptive intermolecular reactive empirical bond-order (AIREBO) potential $^{33}$ obtained from modifications to the Tersoff potential for covalent bonding. Finally, the modified embedded-atom method (MEAM) was created by modifying the EAM to consider the directionality of bonding. The MEAM represents the first interatomic treatment ${ }^{34}$ capable of describing a wide range of elements (e.g., the fcc, bcc, hcp, diamond-structured, and even gaseous elements) using a common formalism.

Here, in the present MD calculations, the interactions between $\mathrm{Cu}-\mathrm{Cu}, \mathrm{H}-\mathrm{H}$, and $\mathrm{Cu}-\mathrm{H}$ atoms were treated on the basis of the BondOrder Potential. As well known, there are four terms associated with BOP. The first term represents the short-range two-body function presenting repulsion between the pairs of the ion cores. The second and third terms account for the $\sigma$ - and $\pi$-bond integrals, while the last term is for the promotion energy of $s p$-valent systems. The interactions between $\mathrm{W}-\mathrm{W}$ atoms were modeled here based on the MEAM. Molecular interactions between $\mathrm{Cu}-\mathrm{W}$ and $\mathrm{H}-\mathrm{W}$ are calculated by the truncated Lennard-Jones (6-12) potential $\Phi_{L J}\left(r_{i j}\right)$, in conjunction with the Lorentz-Berthelot mixing rule. The Lennard-Jones (L-J) potential has the following form:

$$
\Phi_{L J}\left(r_{i j}\right)=4 \varepsilon\left[\left\{\left(\sigma / r_{i j}\right)^{12}-\left(\sigma / r_{i j}\right)^{6}\right\}-\left\{\left(\sigma / r_{c}\right)^{12}-\left(\sigma / r_{c}\right)^{6}\right\}\right] .
$$

In the above expression, the parameter $r_{c}$ denotes a cut-off radius, ${ }^{35}$ while $\varepsilon$ is the binding energy (depth of the potential) and $\sigma$ is the molecular diameter. The values of $\sigma$ for the $\mathrm{Cu}-\mathrm{Cu}, \mathrm{H}-\mathrm{H}$, and $\mathrm{W}-\mathrm{W}$ interactions were taken to be $2.338 \AA$, ${ }^{36} 2.87 \AA$, , and $3.2 \AA$, ${ }^{38}$ respectively; while the values of $\varepsilon$ were found to be $9.43 \mathrm{kcal} / \mathrm{mol}$, ${ }^{36}$ $0.0230 \mathrm{kcal} / \mathrm{mol},{ }^{37}$ and $0.05 \mathrm{kcal} / \mathrm{mol}^{38}{ }^{38}$ respectively. Using these parameters, the Lorentz-Berthelot mixing rule ${ }^{39}$ was employed to calculate the Lennard-Jones parameters for the copper-tungsten and hydrogen-tungsten interactions.

A quick comment regarding the potentials is perhaps in order. Here, we have used a simple approach by invoking the LJ potential in conjunction with the Lorentz-Berthelot mixing rule. Though not the best choice, Lennard-Jones potentials have been used in the past. ${ }^{40-42}$ In addition, various $\mathrm{Cu}-\mathrm{H}$ potentials have been developed based on the embedded-atom method (EAM) formulation ${ }^{43}$ and even the newer bond-order potentials. ${ }^{44}$ However, since the focus of the present simulations is not on the $\mathrm{H}_{2}$ gas phase, nor on clusters, nor on effects on bond strengths due to deformation and fracture, the relatively simple approach can be expected to yield acceptable qualitative trends. Furthermore, the scope here is not to develop accurate interaction potentials.

The MD technique involves solving the equations of motion for interacting particles numerically, subject to appropriate initial and boundary conditions. ${ }^{45}$ The open source LAMMPS code ${ }^{46}$ with its library of potentials was used in the present calculations. Four structures were simulated: (i) a perfect copper lattice with no defects or 
inclusions was used. A face-centered cubic (fcc) structure was used for the copper with a lattice constant of $3.61 \AA$. This was thus effectively a rectangular box representing a slab of copper with atoms lying within it at the lattice points. The top surface above the box was taken to be vacuum. Atoms could escape the top surface, while periodic boundary conditions were applied at the four lateral faces. Specular reflection was applied at the bottom surface. (ii) A uniform copper slab having a small number of hydrogen atoms at interstitial sites is used. This was thus similar to the above geometry but with some hydrogen atoms added to the copper mix. (iii) A copper slab containing a low-angle symmetric tilt grain boundary with hydrogen atoms is included within the volume. The tilt grain boundary was created by defining two crystals having different orientations and deleting the overlapping atoms at the common boundary. Specifically, the $\left[\begin{array}{lll}0 & 0 & 1\end{array}\right]$ crystallographic direction was taken to be parallel to the Z-axis, defined as the tilt axis. With the $\left[\begin{array}{lll}1 & 0 & 0\end{array}\right]$ crystallographic direction aligned with the $\mathrm{X}$-axis, a grain boundary was formed around the middle plane by rotating lattices in the upper half part of the model around the tilt axis by a counterclockwise angle, while those in the lower half part of the model had a clockwise shift. (iv) Finally, to probe possible reductions in outgassing, a composite structure consisting of a $3 \mathrm{~nm}$ tungsten layer was placed on top of the copper surface. A body-centered cubic (bcc) structure was used for the tungsten with a lattice constant of $3.16 \AA$. Hydrogen atoms were initially placed at the copper interstitial sites for this configuration, with escape possible only across the top tungsten surface.

The simulation box with a rectangular cross section for the copper atoms was found to have dimensions of $50 \times 68.5 \times 100 \AA^{3}$ with vacuum above the copper material. Hydrogen atoms were allowed to escape the top surface, while periodic boundary conditions were applied to the other surfaces to simulate infinite extensions. The target temperatures were created by assigning atomic speeds based on a Maxwellian distribution. In all simulations, the starting system configuration was initially relaxed by running the LAMMPS simulations up to $10 \mathrm{~ns}$ to achieve thermodynamic equilibrium and effectively minimize the overall energy. During the thermalization, the NPT (isobaricisothermal) ensemble conditions were used with 29,568 copper atoms. Following relaxation, the operating temperature was maintained at a constant value for each of the simulations, and the NVT (canonical) ensemble conditions were used for the MD simulations. There was no scalar externally applied pressure in these simulations. The Langevin algorithm ${ }^{47}$ was used to control pressure, with a damping parameter $\left(P_{\text {damp }}\right)$ taken to be 0.5 ps. There are several accepted methods for maintaining the system at constant temperature. ${ }^{48,49}$ Here, a Nose-Hoover thermostat ${ }^{50,51}$ was applied, and a time step of $1 \mathrm{fs}$ was used. ${ }^{52}$ Periodic boundary conditions were imposed except at the outer surface for the outgassing evaluations. Here, we do not track the internal stress tensor of a collection of atoms interacting under periodic boundary conditions. Such calculations of internal pressure have been carried out based on Molecular Dynamics simulations for a few decades. ${ }^{53,54}$ However, it was pointed out a decade ago ${ }^{55}$ that the formulations could apply either to nonperiodic cells or to specific pair potentials. A more recent development was a generalization of instantaneous force virial contributions to stress by Thompson et al. ${ }^{56}$ for arbitrary interatomic potentials under periodic conditions.

The diffusion coefficient $(D)$ of hydrogen gas trapped in the copper lattice was obtained by tracking the mean square displacement $\left\langle|r(t)|^{2}\right\rangle$ of all the diffusing hydrogen atoms over a given period of time at each temperature. The mean square displacement (MSD) is related to time-dependent variations in the position for an $\mathrm{N}$-atom system as

$$
\left\langle|r(t)|^{2}\right\rangle=\left\{{ }^{N} \Sigma_{i=1}\left[\boldsymbol{r}_{i}(t)-\boldsymbol{r}_{\mathrm{i}}(0)\right]^{2}\right\} / N
$$

where $\boldsymbol{r}_{i}(t)$ is the position vector of the $i$ th atom at time $t$. The MSD connects the diffusion coefficient through the relationship ${ }^{5}$

$$
D=\left[d\left(\left\langle|r(t)|^{2}\right\rangle\right) / d t\right] / 6 .
$$

Over longer times, the diffusion coefficient saturates to a constant value at the operating temperature. Here, we did not choose to calculate the van Hove distribution function $G(r, t)$ from which the diffusion coefficient could also have been obtained. ${ }^{58,59}$ This is because at shorter times, the self-part of the van Hove correlation function is Gaussian but becomes exponential at longer times. ${ }^{60,61}$ Hence, for simplicity and to avoid dealing with such transitions, the MSD, which scales linearly with time, was chosen instead for evaluating the diffusion coefficient.

\section{RESULTS AND DISCUSSION}

As a validity check of our MD implementation and as a preliminary assessment of our results of hydrogen diffusion in pure copper, MD simulations were first carried out to obtain the temperature-dependent diffusion coefficients. These initial calculations were aimed at facilitating simple comparisons with the published experimental data. To ensure accuracy of results, three sets of simulations were performed for each case. The results were very similar, and the average is reported here. The results of the hydrogen diffusion coefficient obtained from the simulations, along with various temperature-dependent data points reported in the literature, are shown in Fig. 1(a). A fairly good agreement between data and the calculations is evident. The experimental data points include reports by Perkins and Begeal, ${ }^{62}$ Eichenauer and Pebler, ${ }^{63}$ Sakamoto and Takao, ${ }^{64}$ Eichenauer et al., ${ }^{65}$ Ishikawa and McLellan, ${ }^{66}$ and Katz et al. ${ }^{67}$ The diffusion coefficient was obtained from the slope of the mean square displacement vs time, with a representative MD simulation plot at $1000 \mathrm{~K}$ as shown in Fig. 1(b). An Arrhenius type behavior naturally emerged from the calculations, with an activation energy of $0.41 \mathrm{eV}$ and a pre-exponential factor of $6.108 \times 10^{-7} \mathrm{~m}^{2} / \mathrm{s}$, which are comparable to other reports and other materials. ${ }^{21}$

Next, the role of a low-angle grain boundary in the out-diffusion of hydrogen atoms in copper was probed through MD simulations. A copper slab containing a grain boundary with hydrogen atoms initially included as random impurities at interstitial sites uniformly distributed throughout the volume was considered. Initial thermalization and lattice relaxation were carried out for $10 \mathrm{~ns}$. The NPT conditions with periodic boundaries were used to ensure that the hydrogen atoms spread out and were effectively reshuffled within the simulation space. Following this initial phase, an NVT ensemble was used and any hydrogen atom crossing out from a surface was eliminated from the simulation. This provided a count of the outgassing of hydrogen atoms from copper as a function of time. The outflow of hydrogen atoms parallel and perpendicular to the GB plane was tracked.

The results obtained at a temperature of $700 \mathrm{~K}$ are shown in Fig. 2. One hundred fifty hydrogen atoms were inserted into the copper 


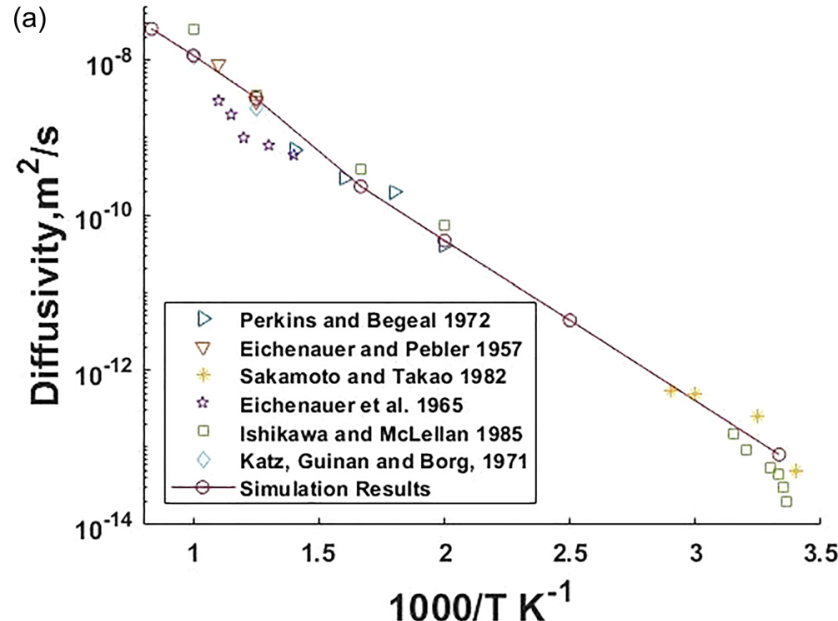

(b)

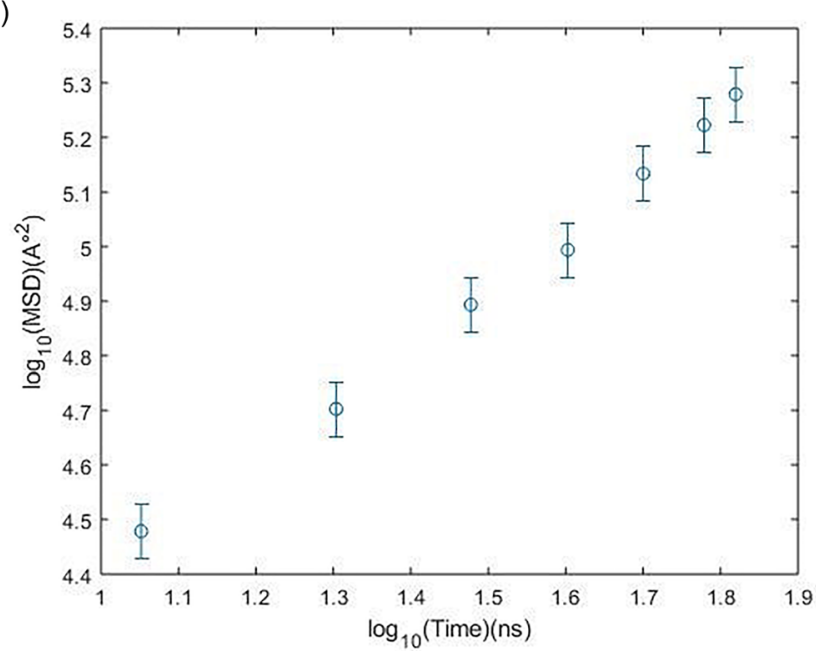

FIG. 1. (a) Comparison between the diffusion coefficient obtained from the simulations and various data points reported in the literature. Good agreement between data and the calculations can be seen. (b) Representative result of the mean square displacement vs time obtained from the MD simulations at $1000 \mathrm{~K}$ for calculating the diffusion coefficient.

lattice to start with. A copper sample without any grain boundary (GB) was included to provide a baseline comparison. The results of Fig. 2 show the structure of losing atoms at the fastest rate for flow parallel to the grain boundary. The number of hydrogen atoms in the copper sample is predicted to reduce from 150 to about 112 in about $10 \mathrm{~ns}$. By comparison, the flow perpendicular to the GB is very slowest, with about 141 of the starting 150 hydrogen atoms remaining in the copper sample. The transport without any grain boundary is seen to lie between these two limits. Roughly, 130 atoms are predicted to remain in copper for the case without a grain boundary after $10 \mathrm{~ns}$. This clearly shows the role played by grain boundaries with regard to transport and outgassing. A grain boundary can effectively provide a pathway to the surface and facilitate outgassing. In the orthogonal direction, the same GB structure acts as a trap and could effectively suppress hydrogen flow. The qualitative trend obtained here in Fig. 2

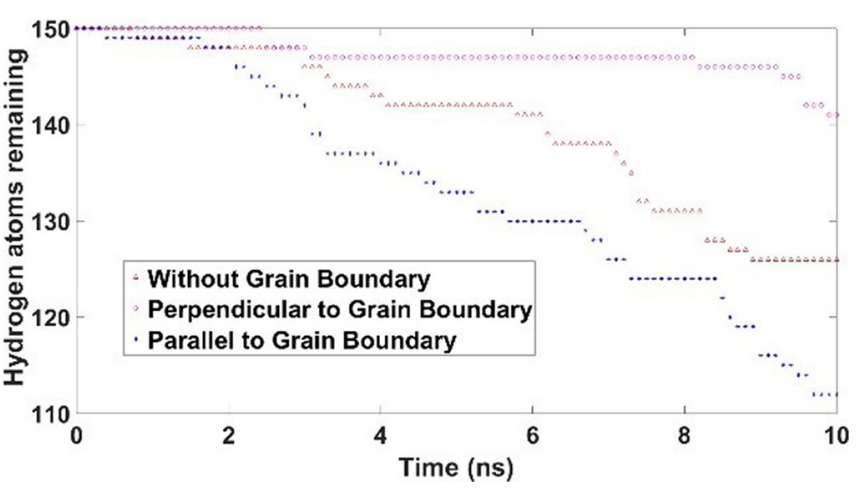

FIG. 2. Simulation results showing the number of hydrogen atoms remaining in a copper slab as a function of time. Cases with and without a grain boundary (GB) were simulated with the out-diffusion in directions both parallel and perpendicular to the GB plane.

is similar to reports that grain boundaries in nuclear fuel materials provide fast transport paths in the absence of any porosity. ${ }^{68,69}$ In the context of nuclear materials, the role of grain boundary diffusion in fission gas release is well known and has been appropriately studied in the past. The atomistic results are in qualitative agreement with the trends from the analytical model first developed by Fisher ${ }^{70}$ who showed diffusion to occur along grain boundaries more rapidly than through the interior of crystals. This model has subsequently been embellished over the years. ${ }^{71}$

Physically, the distortion of atoms in the GB region influences the diffusion of hydrogen atoms. Local deformations and variations within the GB core provide discrete paths for atomic hops. Spatial variability in the local energy near a grain boundary is well known, ${ }^{72}$ and one can expect deviations in the diffusive properties to be strongest at the GB core though the core size variations were not examined here. In the perpendicular direction, the GB acts as a barrier (similar to a mountain range blocking normal flows), leading to very anisotropic transport. However, in an actual sample, diffusion via an interconnected network of grain boundaries can be expected to occur. Since neither the presence of various grain boundaries nor their orientations can be controlled, it becomes impractical to only rely on GBdependent transport to influence outgassing. Instead, a different robust technique for impeding gas outflow becomes necessary.

For completeness, it may be pointed out that two issues that could be important were not probed here. (i) The first involves the role of stress in the sample. For example, the chemical potential can be directly influenced by induced stresses ${ }^{7,74}$ associated with incorporation of solutes such as hydrogen. It was demonstrated that hydrogen diffusivity would increase with the hydrogen content. ${ }^{75,76}$ The origin of this behavior lies in the elastic interactions of the solute with the crystallographic structure. Hydrogen incorporation causes an elastic expansion that changes the chemical potential, thereby aiding diffusion. Though interesting and valid, such aspects will cause only minor changes, and the overall trends obtained would remain. Furthermore, some degree of preheating that is usually carried out before high power operation of such systems will practically guarantee that the hydrogen content and the stress remaining in the electrodes will be quite modest. However, it must be mentioned for completeness and accuracy that though the preheating removes much of the reservoir of gas from the 
bulk material, it does not necessarily solve the issue for stimulated gas desorption. In fact, the preheating process can actually lead to deposition of a few monolayers of gas on the surface, which then becomes available for potential release into the system upon the operation of pulsed power devices. Consequently, many of the high-power, but relatively low energy systems (such as single shot High Power Microwave devices) benefit more from plasma cleaning to remove these adsorbed layers. (ii) The second is the possibility of creating hydrogen bubbles. For example, recent studies ${ }^{7-79}$ suggest that GB can play an important role in the formation of hydrogen bubbles by acting as trapping sites. However, this physics of the nucleation and growth of such bubbles, given the possibility of reduced GB adhesion with the size, ${ }^{23,79}$ remains unresolved.

One possibility to reduce gas out-diffusion might be to add a suitable thin material as a capping overlayer. Toward this goal of mitigating outgassing and to test the effectiveness of an over-coating, a $30 \AA$ thick tungsten layer was added to the simulation structure. The tungsten cap was placed on an open surface, at one end of the simulation box. Tungsten was chosen as a material for the overlayer since diffusion of hydrogen in tungsten has been reported to be fairly low. ${ }^{80}$ To validate and test this hypothesis of low hydrogen diffusivity in tungsten metal, the temperature dependent diffusion coefficient of hydrogen was first calculated in a pure, stand-alone tungsten slab. For this purpose, 50 hydrogen atoms were added to a pure tungsten slab having 7000 metal atoms. The mean square displacement (MSD) vs time was obtained, and the diffusion coefficient was then extracted from the slope. The procedure and calculations were very similar to those for hydrogen in pure copper. Furthermore, a plot of diffusivity vs inverse temperature does not provide much information until the activation energy and pre-exponential factor are extracted. So, for brevity, the diffusion vs inverse temperature results are not shown, and only the time-dependent out-diffusion at a fixed temperature of $1000 \mathrm{~K}$ is given in Fig. 3. However, it must be mentioned that the MD simulations of

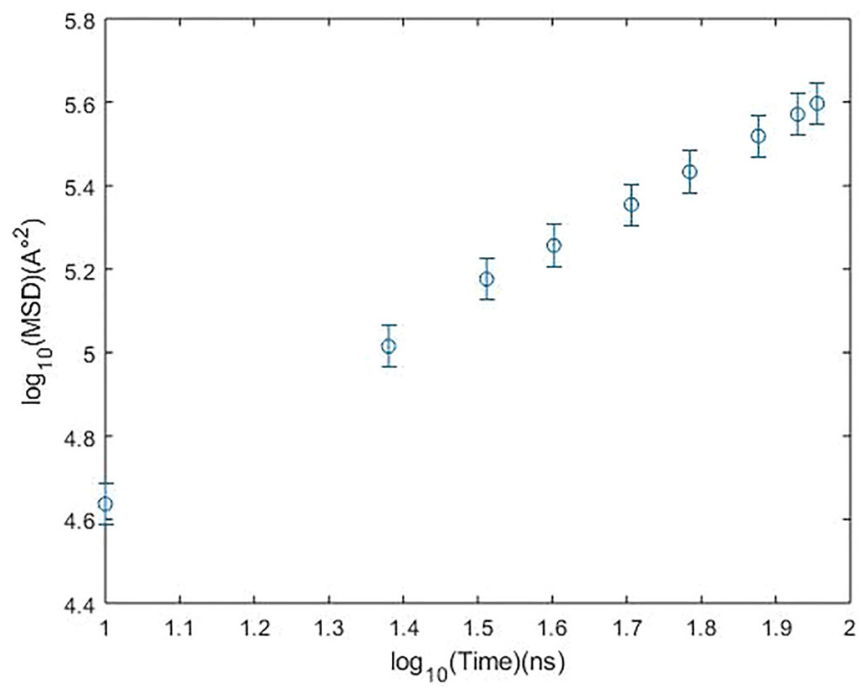

FIG. 3. Mean square displacement (MSD) vs the time plot for hydrogen atoms inserted at random interstitial sites in a tungsten slab. A temperature of $1000 \mathrm{~K}$ was used for the calculations. The diffusion coefficient was obtained from the slope of the above plot. hydrogen diffusion in tungsten at different temperatures yielded an activation energy of $0.42 \mathrm{eV}$ and a pre-exponential factor of 6.918 $\times 10^{-7} \mathrm{~m}^{2} / \mathrm{s}$. The activation energy was similar to that reported by Frauenfelder. ${ }^{81}$ From the results of Fig. 3, the value of the diffusion coefficient for hydrogen in tungsten was calculated to be $5.311 \times 10^{-9} \mathrm{~m}^{2} / \mathrm{s}$. The reported value in the literature for hydrogen diffusion in tungsten is roughly $5.484 \times 10^{-9} \mathrm{~m}^{2} / \mathrm{s}^{80}$ at $1000 \mathrm{~K}$. Thus, these results for hydrogen diffusion in tungsten not only point the validity of the calculations but also underscore the much lower diffusivity in this material as compared to a copper host.

Finally, calculations of hydrogen outgassing from copper in the presence of a tungsten cap-layer were carried out. As shown in Fig. 2, a low-angle grain boundary was assumed in the copper. As before, the system was thermalized using the NPT ensemble for $10 \mathrm{~ns}$. After shuffling all the atoms (i.e., hydrogen, copper, and tungsten) during thermalization, the actual simulations were then performed for $30 \mathrm{~ns}$. The comparison of outgassing with and without the presence of a tungsten layer is shown in Fig. 4 at a temperature of $700 \mathrm{~K}$. In the results, the number of hydrogen atoms is predicted to reduce to around 110 from an initial number of 150, in about $10 \mathrm{~ns}$. Rather than comparing diffusion coefficients, the time-dependent populations escaping the metal system provide a more convenient and direct basis for comparison. In these simulations, there were no surface defects or trace impurities that might lead to surface recombination or trapping. With the added tungsten layer, the outgassing rate is seen to be much lower with about 142 hydrogen atoms predicted to remain in the simulation box even after 30 ns. In Fig. 4, over a duration of $10 \mathrm{~ns}$, about 39 hydrogen atoms are predicted to leave the pure, homogeneous copper slab; while only 1 hydrogen atom escapes the metallic system with the tungsten cap layer. This is almost a factor of 40 reduction in the outgassing rate. So it is quite evident that the tungsten capping layer can work as a material based strategy to effectively mitigate outgassing.

The above calculations and results could form the basis for predictive analysis of gas emissions per unit area. For example, temperature dependent emission rates (or alternatively average lifetimes of the atomic gas) could be obtained from plots of the time-dependent decrease in the number of hydrogen atoms within copper at different temperatures. By knowing the surface area of the simulation box, the outflow rates per unit area could be estimated. For statistical accuracy though, averages over numerous simulation runs would be needed.

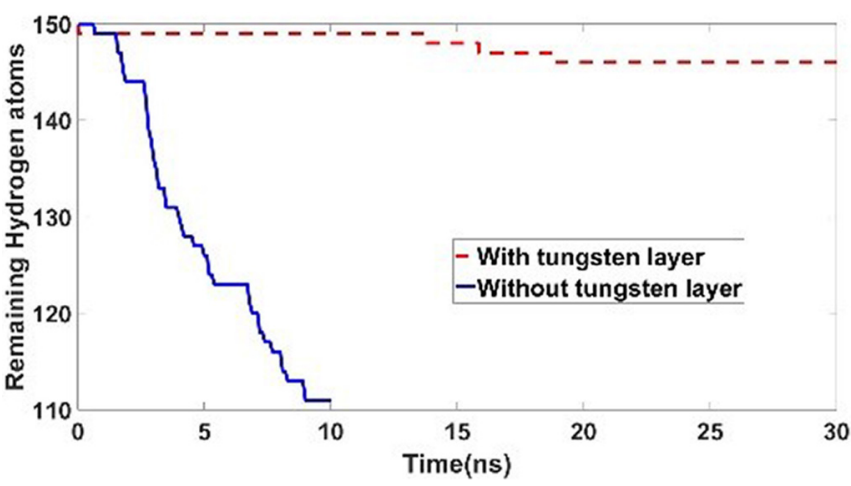

FIG. 4. Comparison of outgassing rates with and without the tungsten over-layer 
A practical validity test might then be to compare the predicted emission of hydrogen gas with the measured increase in pressure within the test chambers. In these cases, the simulations would have to specifically assign the gas atoms within the top few monolayers of the surface.

Furthermore, an aspect, not addressed here, but would be important in the context of charged particle incidence upon electrodes in pulsed power systems, would be the time dependent changes in temperature and associated spatial gradients. The latter could add a thermo-diffusive contribution to gas transport. In any event, a detailed analysis would require a three-step process of: (i) initial calculations of the deposited energy from the incident particles, possibly through Monte Carlo calculations with energy-dependent Stopping Power and Mean Free Paths, (ii) solutions of heat transport to obtain the evolutionary spatially variable temperature profiles, and (iii) use of the spatially dependent temperatures thus obtained as inputs in the MD technique to naturally include thermal gradients.

\section{CONCLUSIONS}

Outgassing is typically the first stage of possible plasma formation in a vacuum and can lead to various effects such as breakdown, surface flashover, anode-cathode gap closure, and pulse shortening in high power systems. It is therefore important to understand the outgassing process and even more critical to device ways for its mitigation. Here, preliminary simulation results of hydrogen diffusion and outgassing in a representative metal (such as copper) were obtained based on the molecular dynamics technique. The calculated temperature dependent diffusion coefficient values agreed with experimental reports in the literature over a range spanning from $300 \mathrm{~K}$ to $1330 \mathrm{~K}$. The temperature variation could be important in the context of power devices and vacuum nanoelectronics which tend to have high electric field environments or might be subjected to external radiation, both of which can lead to localized heating. The role of heating in enhancing diffusive outflow by orders of magnitude has been demonstrated here.

Simulations with and without a grain boundary were also carried out to probe their role in hydrogen outgassing from metals. The central result was increased outdiffusion in the presence of a grain boundary (GB) along parallel planes due to an effective channeling effect. The most likely reason would be associated with distortion of atoms in the GB region, with the local deformations providing alternate paths for atomic hops within the GB core. The diffusion was shown to be anisotropic, with the increase parallel to the GB plane and reduction in the normal direction. However, in an actual sample, diffusion via an interconnected network of grain boundaries can be expected to occur. Since neither the presence of grain boundaries nor their orientations can be controlled, it becomes impractical to rely on GB-dependent transport as a means to tailor or influence outgassing. Instead, an alternative based on using a cap layer composed of a thin metallic coating with low material diffusion was examined here as a possible solution. A $30 \AA$ thick tungsten layer was added over the copper surface to test the outflow. The results were promising and demonstrated that the tungsten capping layer can work as a material-based strategy to effectively mitigate outgassing. For durability and to avoid pinholes or damage, thicker layers would be more useful practically.

\section{ACKNOWLEDGMENTS}

We thank unknown reviewers for some useful comments. This work was supported in part by grants from the Office of Naval
Research (N00014-18-1-2382) and the Air Force Office of Scientific Research (FA9550-19-1-0056).

\section{REFERENCES}

${ }^{\mathbf{1}}$ R. L. Ives, IEEE Trans. Plasma Sci. 32, 1277 (2004).

${ }^{2}$ R. J. Barker and E. Schamiloglu, High-Power Microwave Sources and Technologies (Wiley-IEEE Press, New York, 2001).

${ }^{3}$ J. H. Booske, R. J. Dobbs, C. D. Joye, C. L. Kory, G. R. Neil, G. S. Park, J. Park, and R. J. Temkin, IEEE Trans. Terahertz Sci. Technol. 1, 54 (2011).

${ }^{4}$ S. D. Korovin, V. V. Rostov, S. D. Polevin, I. V. Pegel, E. Schamiloglu, M. I. Fuks, and R. J. Barker, Proc. IEEE 92, 1082 (2004).

${ }^{5}$ D. Keefe, Part. Accel. 11, 187 (1981).

${ }^{6}$ R. S. Craxton, K. S. Anderson, T. R. Boehly, V. N. Goncharov, D. R. Harding, J. P. Knauer, R. L. McCrory, P. W. McKenty, D. D. Meyerhofer, J. F. Myatt, A. J. Schmitt, J. D. Sethian, R. W. Short, S. Skupsky, W. Theobald, W. L. Kruer, K. Tanaka, R. Betti, T. J. B. Collins, J. A. Delettrez, S. X. Hu, J. A. Marozas, A. V. Maximov, D. T. Michel, P. B. Radha, S. P. Regan, T. C. Sangster, W. Seka, A. A. Solodov, J. M. Soures, C. Stoeckl, and J. D. Zuegel, Phys. Plasmas 22, 110501 (2015).

${ }^{7}$ X. Qiu and R. P. Joshi, Phys. Plasmas 25, 022109 (2018).

${ }^{8}$ A. Majzoobi, R. P. Joshi, A. A. Neuber, and J. Dickens, AIP Adv. 5, 127237 (2015).

${ }^{9}$ A. Roy, R. Menon, S. Mitra, S. Kumar, V. Sharma, K. V. Nagesh, K. C. Mittal, and D. P. Chakravarthy, Phys. Plasmas 16, 053103 (2009).

${ }^{10}$ J. Benford and G. Benford, IEEE Trans. Plasma Sci. 25, 311 (1997).

${ }^{11}$ E. E. Kunhardt, S. Popovic, and J. Bentson, IEEE Trans. Plasma Sci. 23, 970 (1995).

${ }^{12}$ A. A. Neuber, M. Butcher, H. Krompholz, L. L. Hatfield, and M. Kristiansen, IEEE Trans. Plasma Sci. 28, 1593 (2000).

${ }^{13}$ N. M. Jordan, G. B. Greening, B. W. Hoff, S. S. Maestas, S. C. Exelby, and R. M. Gilgenbach, IEEE Trans. Plasma Sci. 44, 1258 (2016).

${ }^{14}$ M. Takeda, H. Kurisu, S. Yamamoto, H. Nakagawa, and K. Ishizawa, Appl. Surf. Sci. 258, 1405 (2011).

${ }^{15}$ M. D. Haworth, K. L. Cartwright, J. W. Luginsland, D. A. Shiffler, and R. J. Umstattd, IEEE Trans. Plasma Sci. 30, 992 (2002).

${ }^{16}$ A. A. Avdienko and A. V. Kiselev, Sov. Phys. Tech. Phys. 12, 381 (1967).

${ }^{17}$ M. Akahane, Y. Ohki, D. Ito, and K. Yahagi, Electr. Eng. Jpn. 94, 1 (1974).

${ }^{18}$ J. Zheng, X. Liu, P. Xu, P. Liu, Y. Zhao, and J. Yang, Int. J. Hydrogen Energy 37, 1048 (2012).

${ }^{19}$ M. S. Daw and M. I. Baskes, Phys. Rev. Lett. 50, 1285 (1983).

${ }^{20}$ H. L. Hou, Z. Q. Li, Y. J. Wang, and Q. Guan, Chin. J. Nonferrous Met. 13, 533 (2003).

${ }^{21}$ X. W. Zhou, F. El-Gabaly, V. Stavila, and M. D. Allendorf, J. Phys. Chem. C 120, 7500 (2016).

${ }^{22}$ B. Szpunar, L. J. Lewis, I. Swainson, and U. Erb, Phys. Rev. B 60, 10107 (1999).

${ }^{23}$ H. B. Zhou, Y. L. Liu, S. Jin, Y. Zhang, G. N. Luo, and G. H. Lu, Nucl. Fusion 50, 025016 (2010).

${ }^{24}$ M. S. Daw, S. M. Foiles, and M. I. Baskes, Mater. Sci. Rep. 9, 251 (1993).

${ }^{25}$ B. J. Lee, J. Phase Equilib. Diffus. 30, 509 (2009).

${ }^{26}$ M. S. Daw and M. I. Baskes, Phys. Rev. B 29, 6443 (1984).

${ }^{27}$ M. W. Finnis and J. E. Sinclair, Philos. Mag. A 50, 45 (1984).

${ }^{28}$ F. Ercolessi, E. Tosatti, and M. Parrinello, Phys. Rev. Lett. 57, 719 (1986)

${ }^{29}$ J. Tersoff, Phys. Rev. B 37, 6991 (1988).

${ }^{30}$ V. Rosato, M. Guillope, and B. Legrand, Philos. Mag. A 59, 321 (1989).

${ }^{31} \mathrm{~J}$. Tersoff, Phys. Rev. Lett. 56, 632 (1986).

${ }^{32}$ D. G. Pettifor and I. I. Oleinik, Phys. Rev. B. 59, 8487 (1999).

${ }^{33}$ S. J. Stuart, A. B. Tutein, and J. A. Harrison, J. Chem. Phys. 112, 6472 (2000).

${ }^{34}$ M. I. Baskes, Phys. Rev. B 46, 2727 (1992).

${ }^{35}$ M. P. Allen and D. J. Tildesley, Computer Simulation of Liquids (Oxford, Clarendon Press, Oxford, 1987).

${ }^{36}$ Q. C. Hsu, C. D. Wu, and T. H. Fang, Comput. Mater. Sci. 34, 314 (2005).

${ }^{37}$ Y. Saito, N. Sasaki, and T. Komatsu, IEEE Trans. Magn. 48, 2009 (2012).

${ }^{38}$ M. Wang, K. M. Liechti, V. Srinivasan, J. M. White, P. J. Rossky, and M. T. Stone, J. Appl. Mech. 73, 769 (2006).

${ }^{39}$ P. M. Agrawal, B. M. Rice, and D. L. Thompson, Surf. Sci. 515, 21 (2002). 
${ }^{40}$ For example, P. B. Lloyd, J. W. Kress, and B. J. Tatarchuk, Appl. Surf. Sci. 119, 275 (1997).

${ }^{41}$ A. A. Ermolenko, G. V. Kornich, and G. Betz, Bull. Russ. Acad. Sci.: Phys. 74, 114 (2010).

${ }^{42}$ A. Fornit, G. Wiesenekkert, E. J. Baerendst, and G. F. Tantardin, I. Phys: Condens. Matter 7, 7195 (1995).

${ }^{43}$ Q. Sun, J. Xie, and T. Zhang, Surf. Sci. 338, 11 (1995).

${ }^{44}$ X. W. Zhou, D. K. Ward, M. Foster, and J. A. Zimmerman, J. Mater. Sci. 50, 2859 (2015)

${ }^{45}$ B. J. Alder and T. E. Wainright, J. Chem. Phys. 27, 1208 (1957).

${ }^{46}$ S. Plimpton, J. Comput. Phys. 117, 1 (1995).

${ }^{47}$ T. Schneider and E. Stoll, Phys. Rev. B 17, 1302 (1978).

${ }^{48}$ H. C. Andersen, J. Chem. Phys. 72, 2384 (1980).

${ }^{49}$ H. J. C. Berendsen, J. P. M. Postma, W. F. van Gunsteren, A. DiNola, and J. R. Haak, J. Chem. Phys. 81, 3684 (1984).

${ }^{50}$ S. Nose, J. Chem. Phys. 81, 511 (1984)

${ }^{51}$ S. Nose, Mol. Phys. 100, 191 (2002).

${ }^{52}$ Z. Zhang, M. Giesselmann, J. Mankowski, J. Dickens, A. Neuber, and R. P. Joshi, J. Phys. D 50, 185202 (2017).

${ }^{53}$ M. P. Allen and D. J. Tildesley, Computer Simulation of Liquids (Clarendon, Oxford, 1991)

${ }^{54}$ J. J. Erpenbeck and W. W. Wood, in Modern Theoretical Chemistry, Statistical Mechanics, Part B: Time Dependent Processes, edited by B. J. Berne (Plenum, New York, 1977).

${ }^{55}$ M. J. Louwerse and E. J. Baerends, Chem. Phys. Lett. 421, 138 (2006).

${ }^{56}$ A. P. Thompson, S. J. Plimpton, and W. Mattson, J. Chem. Phys. 131, 154107 (2009).

${ }^{57}$ R. Kubo, Rep. Prog. Phys. 29, 255 (1966).

${ }^{58}$ J. P. Hansen and I. R. McDonald, Theory of Simple Liquids, 3rd ed. (Academic Press, London, 2006).

${ }^{59}$ L. van Hove, Phys. Rev. 95, 249 (1954).
${ }^{60}$ B. Wang, J. Kuo, S. C. Bae, and S. Granick, Nat. Mater. 11, 481 (2012).

${ }^{61}$ J. Kim, C. Kim, and B. J. Sung, Phys. Rev. Lett. 110, 047801 (2013).

${ }^{62}$ W. G. Perkins and D. R. Begeal, Ber. Bunsengesellschaft 76, 863 (1972).

${ }^{63}$ W. Eichenauer and A. Pebler, Z. Metall. 48, 373 (1957).

${ }^{64}$ Y. Sakamoto and K. Takao, J. Jpn. Inst. Met. 46, 285 (1982).

${ }^{65}$ W. Eichenauer, W. Löser, and H. Witte, Z. Metall. 56, 287 (1965).

${ }^{66}$ T. Ishikawa and R. B. McLellan, J. Phys. Chem. Solids 46, 445 (1985).

${ }^{67}$ L. Katz, M. Guinan, and R. J. Borg, Phys. Rev. B 4, 330 (1971).

${ }^{68}$ J. A. Turnbull, C. A. Friskney, F. A. Johnson, A. J. Walter, and J. R. Findlay, J. Nucl. Mater. 67, 301 (1977).

${ }^{69}$ D. R. Olander and P. van Uffelen, J. Nucl. Mater. 288, 137 (2001).

${ }^{70}$ J. C. Fisher, J. Appl. Phys. 22, 74 (1951).

${ }^{71}$ For example, I. Kaur, Y. Mishin, and W. Gust, Fundamentals of Grain and Interphase Boundary Diffusion (Wiley, New York, 1995).

${ }^{72}$ A. Suzuki and Y. Mishin, Interface Sci. 11, 131 (2003).

${ }^{73}$ P. H. Dederichs and K. Schroeder, Phys. Rev. B 17, 2524 (1978).

${ }^{74}$ H. Haftbaradaran, J. Song, W. A. Curtin, and H. J. Gao, Power Sources 196, 361 (2011).

${ }^{75}$ J. Li, A. Oudriss, A. Metsue, J. Bouhattate, and X. Feaugas, Sci. Rep. 7, 45041 (2017).

${ }^{76}$ X. Zhou, D. Marchand, D. L. McDowell, T. Zhu, and J. Song, Phys. Rev. Lett. 116, 075502 (2016).

${ }^{77}$ T. Shimada, H. Kikuchi, Y. Ueda, A. Sagara, and M. Nishikawa, J. Nucl. Mater. 313-316, 204 (2003).

${ }^{78}$ T. Funabiki, T. Shimada, Y. Ueda, and M. Nishikawa, J. Nucl. Mater. 329-333, 780 (2004)

${ }^{79}$ N. Enomoto, S. Muto, T. Tanabe, J. W. Davis, and A. A. Haasz, J. Nucl. Mater. 385, 606 (2009).

${ }^{80} \mathrm{~K}$. Heinola and T. Ahlgren, J. Appl. Phys. 107, 113531 (2010).

${ }^{81}$ R. Frauenfelder, J. Vac. Sci. Technol. 6, 388 (1969). 